\title{
Renforcer l'attractivité de la profession de médecin
}

\section{Comité central de la FMH}

La part des médecins qui n'exercent plus auprès des patients oscille entre 8,4 et $12,9 \%$. En d'autres termes, près de 80 médecins en âge de travailler quittent chaque année la profession, tandis que 800 autres terminent leur formation. Pour la grande majorité de ceux qui tournent le dos à la profession, cette décision est définitive. Selon une étude réalisée récemment, seul un médecin concerné sur 10 (9\%) envisage de manière plutôt probable, voire très probable de reprendre une activité médicale. Les raisons invoquées sont le plus souvent étroitement liées aux conditions de travail.

\section{Situation initiale}

La question de savoir combien de médecins choisissent de ne pas exercer d'activité médicale au terme de leur formation a été soulevée à de nombreuses reprises par les médias, à l'instar d'un article paru dans la NZZ am Sonntag ${ }^{1}$ qui esquisse des solutions pour réduire le nombre de médecins qui tournent le dos à la profession. Un point fait l'unanimité: il faut autant que possible éviter que les médecins abandonnent la profession, notamment en raison de la pénurie croissante qui s'annonce.

Il est indéniable aussi que les qualifications médicales sont utiles, voire requises pour exercer une activité autre que médecin traitant, par exemple en médecine des assurances, dans l'administration ou la recherche. L'étude mandatée par la FMH et l'ASMAC ${ }^{2}$ recense cependant entre 8,4 et $12,9 \%$ des médecins qui n'exercent plus directement auprès des patients; un taux équivalant à près de 80 médecins sur l'ensemble des diplômés annuels en médecine humaine. La décision de ne plus être en contact direct avec les patients est définitive pour la majorité d'entre eux. En effet, selon cette même étude, seul un médecin sur 10 juge probable, voire très probable de reprendre son activité médicale.

Les raisons invoquées sont le plus souvent étroitement liées aux conditions de travail dans le domaine des soins, avec au premier plan le taux d'activité et les horaires de travail, considérés par un médecin sur trois comme l'une des trois raisons principales de sa ré- orientation. Plus d'une personne sur cinq mentionne également la compatibilité de l'activité médicale avec la garde des enfants, et le contenu du travail. Le troisième facteur, à savoir le niveau d'exigence de la profession, qui concerne l'activité à proprement parler, précède d'autres raisons comme la santé, la volonté de se réorienter professionnellement et la retraite.

D'autres facteurs favorisent l'abandon de la profession de médecin:

- Les médecins qui arrêtent d'exercer ont une vision plus critique de la profession que leurs confrères actifs dans le domaine des soins.

- Les médecins qui ne traitent plus de patients le font en connaissance de cause, c'est-à-dire qu'ils connaissent déjà les défis de la profession au terme de leurs études.

- L'argument d'une situation médicale insatisfaisante n'a pas plus de poids que les autres possibilités professionnelles intéressantes qui se sont proposées.

\section{Les arguments}

Pour la FMH et l'ASMAC, l'accent doit être mis sur les mesures suivantes afin d'encourager les médecins à poursuivre leur activité auprès des patients:

- Mise en œuvre des dispositions légales en vigueur Tous les médecins-assistants sont soumis à la loi sur le travail depuis le $1^{\text {er }}$ janvier 2005. Cette loi n'est malheureusement pas toujours appliquée dans de nombreux endroits. 
- Création de conditions de travail adaptées au mode de vie actuel

La FMH et l'ASMAC s'investissent pour des conditions de travail adaptées aux attentes d'aujourd'hui et pour une meilleure conciliation entre vie privée et vie professionnelle. Taux d'activité adapté, emplois du temps modulables et réguliers, postes à temps partiel et enfin planning établis en concertation avec les médecins sont des aspects importants pour concilier vie professionnelle et familiale. Dans ce contexte, il convient également de créer davantage de places de crèches avec horaire élargi, à proximité du lieu de travail.

- Réduction des tâches administratives

De nombreuses études montrent que les tâches administratives des médecins ne cessent d'augmenter aussi bien à l'hôpital qu'au cabinet. L'attractivité de la profession peut être accrue en remettant au centre le temps consacré au patient; les médecins pourront à nouveau se concentrer sur leur activité principale, ce qui redonne du sens au contenu de leur travail et améliore la satisfaction personnelle.
- Sensibilisation des médecins tout au long de leur carrière

Les médecins sont très fortement sollicités dans l'exercice de leur profession, ce qui affecte parfois leur santé physique et psychique. Les futurs médecins doivent donc être préparés aux défis qui les attendent au cours de leur formation, et cette question doit également être traitée activement pendant la formation postgraduée et continue.

\section{Nos exigences}

1. Création de postes de travail à temps partiel dans tous les secteurs et à tous les échelons hiérarchiques

2. Réduction des tâches administratives

3. Création de crèches et garde d'enfants adaptée aux horaires (irréguliers)

4. Respect des dispositions légales en vigueur

5. Aide aux médecins lors de l'ouverture ou de la reprise d'un cabinet

6. Contenus de travail centrés sur les patients et reconnaissance du travail fourni

7. Promotion de la santé et prévention pour les médecins 